\title{
Critical Findings Reporting in Cranial CT Scans of Patients in a Tertiary Government Hospital Setting. A Pilot Study.
}

\author{
Andro Reginald L. Licaros' ${ }^{1}$ [MD] \\ ORCID: 0000-0001-9135-8929 \\ Johanna Patricia A. Cañal ${ }^{1}$, [MD] \\ ORCID: 0000-0001-5407-1478 \\ ${ }^{1}$ University of the Philippines - Philippine General Hos- \\ pital, Department of Radiology, Manila, Philippines
}

*Corresponding author: Andro Reginald L. Licaros, MD University of the Philippines - Philippine General Hospital, Department of Radiology, Manila, Philippines: Phone: +632554-8400 local 3104

e-mail: androlicaros@gmail.com

\section{Johanna Patricia A. Cañal, MD, MHA, FPCR}

University of the Philippines - Philippine General Hospital, Department of Radiology, Manila, Philippines: Phone: +632554-8400 local 3104

e-mail:jdadevosocanal@up.edu.ph

\section{nee) ABSTRACT Cen}

Objective: Effective communication among health care providers within an institution is vital for ensuring patient safety. A radiologist's competence in identifying critical findings is just as important as being able to relay this information to the primary provider in a timely manner so that appropriate interventions can be initiated in life-threatening emergencies. This study aims to obtain baseline data regarding effective communication of critical findings between radiology and the primary health care providers.

Materials and Methods: Based on the Massachusetts's Coalition for Prevention of Medical Errors safe practice recommendations, a communication protocol was developed. Certain selected cranial critical findings (new hemorrhage, new herniation, acute brain edema, acute stroke and findings suggestive of meningitis or abscess) were used in the critical findings protocol. Total communication time in the relay of critical findings included time from CT scan order to scan, time from scan to interpretation and time from interpretation to relay. All these times were recorded and compared to the standard 60 minutes using percent compliance, mean time, median time and standard deviation.

Results: Seventy-nine (79) critical findings were recorded and relayed in a 30-day period. There was $100 \%$ (79/79) compliance with the relay of critical findings to the primary care team and $97.5 \%$ (77/79) success rate in direct communication of critical findings within 60 minutes of identification. The mean time from CT scan order to scan was $155.09( \pm 134.43)$ minutes, mean time from scan to interpretation was $46.54( \pm 44.01)$ minutes, and mean time from interpretation to relay was $9.13( \pm 12.04)$ minutes. The average time elapsed from CT study acquisition to direct notification was $57.0( \pm 45.80)$ minutes.

Conclusion: Effective communication of critical findings using a protocol adopted from set standards of safe practice recommendations is achievable in our institution. The proposed protocol exhibited compliance to and performed well against established benchmarks. The timely identification and delivery of critical findings to the primary care team is central to patient management and should be practiced in our setting.

Keywords: Critical finding, effective communication, cranial CT, standards

Received: 12 March 2020, Accepted: 23 April 2020,

Published online: 20 May 2020

\section{INTRODUCTION}

Clear and effective communication between physicians is of utmost importance in delivering quality patient care - that is, optimum care in a timely manner. It is well studied that miscommunication is a leading cause of inadvertent patient care and/or medical errors, making it essential to establish clear lines of communication between physicians $[1,2]$. In research done by Woolfe et. al., approximately $80 \%$ of errors stemming from miscommunication between medical practitioners led to a 
cascade propagating diagnostic and treatment mistakes [3].

It is, therefore, crucial that radiologists not only interpret images accurately but also effectively communicate these findings to those responsible for treatment decisions. The American College of Radiology practice parameters for communicating diagnostic imaging findings stress that communication of information is only as effective as the system that conveys the information [4]. A well-reviewed and tested communication system is critical in providing the standard of care in any medical care facility. For this reason, The Joint Commission in 2011 placed communication of critical results as a priority goal for its National Patient Safety Standards in the United States [5].

The Joint Commission (TJC) and its international branch, The Joint Commission International (TJCl), are independent, not-for-profit organizations that accredit and certify health care organizations and programs across the globe. Accreditation and certification by The Commission are recognized as a symbol of quality that reflects commitment to meeting certain performance standards, including patient safety [6]. Currently, the Philippine General Hospital is not a TJCl-accredited hospital.

A critical test result is defined as a finding that requires direct notification of a member of the patient's care team, including findings that could result in mortality or significant morbidity if appropriate steps are not undertaken [7]. In the United States, TJC trusts each hospital's leadership to approve and validate their own definition of critical test results $[7,8]$. In the Philippine General Hospital, no vetted critical radiology findings list or protocol for neuroradiology or cranial CT findings exists. Upon discovery, the communication of these findings is inconsistent.

Using a protocol adopted and modified from the Massachusetts Coalition for the Prevention of Medical Errors' safe practice recommendations for the communication of critical radiology findings published in TJC's Journal on Quality and Patient Safety, and a study done by Viertel et. al. in reporting critical findings in neuroradiology, we selected the following as critical cranial findings requiring immediate interruptive notification: new cerebral hemorrhage, new herniation, acute brain edema, acute stroke and findings suggestive of meningitis or abscess [8].

In this study, we classified our critical neuroradiology findings similar to the Brigham and Women's Hospital (BWH in Boston, Massachusetts) Department of Radiology version of the Coalition's recommendations. BWH has a color-coded system consisting of red, orange and yellow alerts [9]. The systems require caregiver notification in $<60 \mathrm{~min}$ utes, $<3$ hours and $<15$ days respectively. Red values are those considered to be potentially life-threatening to the patient unless treatment is initiated immediately and should be communicated within 60 minutes of detection [9]. We considered the selected critical findings as red values and used its set time ( $<60$ minutes) as the standard for measuring the performance of our communications protocol. The primary objective of this paper was to obtain baseline data regarding reporting critical radiology findings within a tertiary government hospital by measuring performance and compliance against standards using the protocol adopted from the Joint Commission and the Massachusetts Coalition for prevention of medical errors. Specifically, this study aimed to determine the relevant time points involved in the communication of critical findings and identify reasons for possible delay and to identify the possible sources of communication breakdown in the relay of critical findings between different services within the Philippine General Hospital.

\section{MATERIALS and METHODS}

This study utilized an observational design. The Philippine General Hospital Department of Radiology served as study site where the proposed protocol for relaying critical findings was implemented for one month. The prospective study ran from February 4, 2019 until March 5, 2019.

Inclusion criteria involved PGH radiology residents responsible for $\mathrm{CT}$ studies (CT team) and non-radiology physicians requesting cranial CT studies. In the 
PGH, a CT team is composed of at least 6 residents at any given month- 5 senior residents and at least one junior resident. Radiology residents part of the $\mathrm{CT}$ team during the study was thoroughly briefed on the critical findings protocol and the research explained to them by the principal investigator.

Non-radiology physicians with patients meeting the criteria for activation of the protocol were approached and informed of the study by a designated $\mathrm{CT}$ team member when these physicians have their CT request approved. Consent forms was available to both radiology and non-radiology physicians and was secured from those willing to participate.

All PGH adult patients at least 18 years old, from the Emergency Department, the intensive care units and the floors, scanned during the investigation period with identified critical findings on new or follow-up cranial CT scans were included. CT critical findings were defined as:

1. New hemorrhage (epidural, subdural, intraparenchymal, subarachnoid, intraventricular and intramedullary hemorrhage)

2. New herniation (subfalcine, transalar, transtentorial and tonsillar herniations)

3. Acute brain edema defined as new onset of diffuse effacement of cerebral and cerebellar sulcal effacement.

4. Acute stroke defined as stroke-like symptoms with an onset more than 6 hours but less than 3 days with one or more of the following $C T$ findings: parenchymal hypodensity, hyperdense vessel sign, loss of gray matter-white matter distinction and gyral swelling or sulcal effacement.

5. Findings suggestive of meningitis (on contrast studies, diffuse or focal undue leptomeningeal enhancement) or abscess (any focus suggestive of a rim-enhancing lesion on contrast studies) $[8,10]$.

All CT patients included in the Brain Attack Protocol of PGH, pediatric patients and those scanned from the out-patient department were excluded from this study.

A critical findings communication protocol was adopted modelling that of the Massachusetts's Coalition for the Prevention of Medical Errors (Figure 1). The principal investigator thoroughly briefed the
Radiology residents and fellows who were part of the $\mathrm{CT}$ team during the study period on the critical findings protocol.

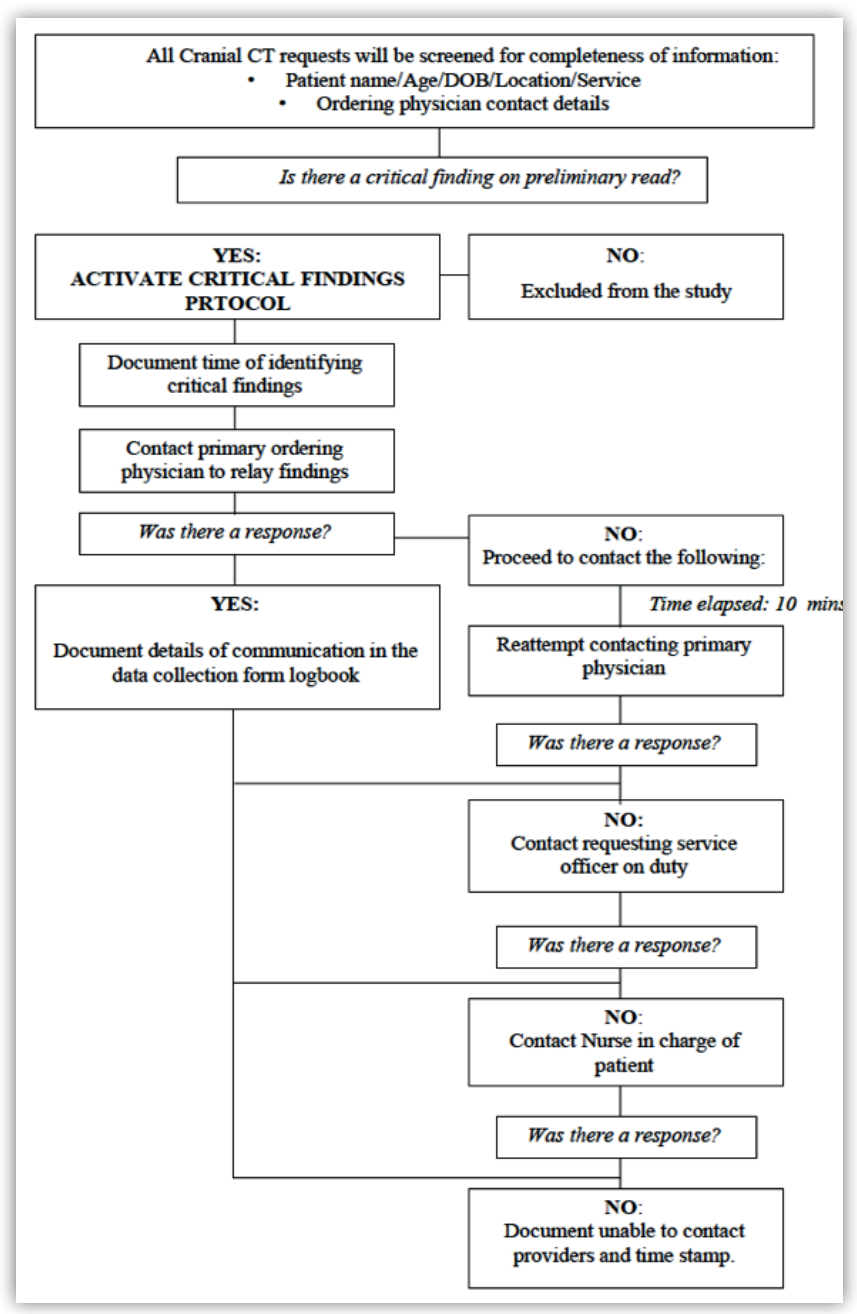

Figure 1. Protocol for Relaying Critical Radiology Findings

In the Philippine General Hospital, all CT scan requests are screened by a diagnostic radiology resident before the study is done. All cranial $\mathrm{CT}$ request forms were verified for completion by the $\mathrm{CT}$ resident. To be considered complete, all the blanks of the request form must be filled in. The data required include the contact details of the requesting physician or personnel on duty and patient identifying details including name, medical record number, age, date of birth and current patient location. Identification of a critical finding in the preliminary CT findings activated the proposed protocol. CT findings were evaluated by the senior resident. A designated senior $\mathrm{CT}$ resident for the day/shift 
was in charge of communicating the critical findings and documenting the process. The communication protocol entailed making a call to the ordering physician to relay the critical findings within 60 minutes of detection or identification. If there was no response after 10 minutes, a second attempt was made to contact the ordering physician. Failure of response led to calling the physician/duty mate of the ordering physician. No answer led to a final call to the nurse-in-charge of the patient to communicate the critical finding to the primary care team members. A time log for every call attempt was documented until results were successfully communicated, which ended the protocol. The possible reasons for delays in communication were logged in the provided form.

A log book was provided for obtaining the required information \& record keeping. Time records were obtained from the synchronized digital clock of the CT console and mobile phone used to communicate the finding. During the study period, the usual process and flow of patient CT scanning was unchanged (Figure 2).

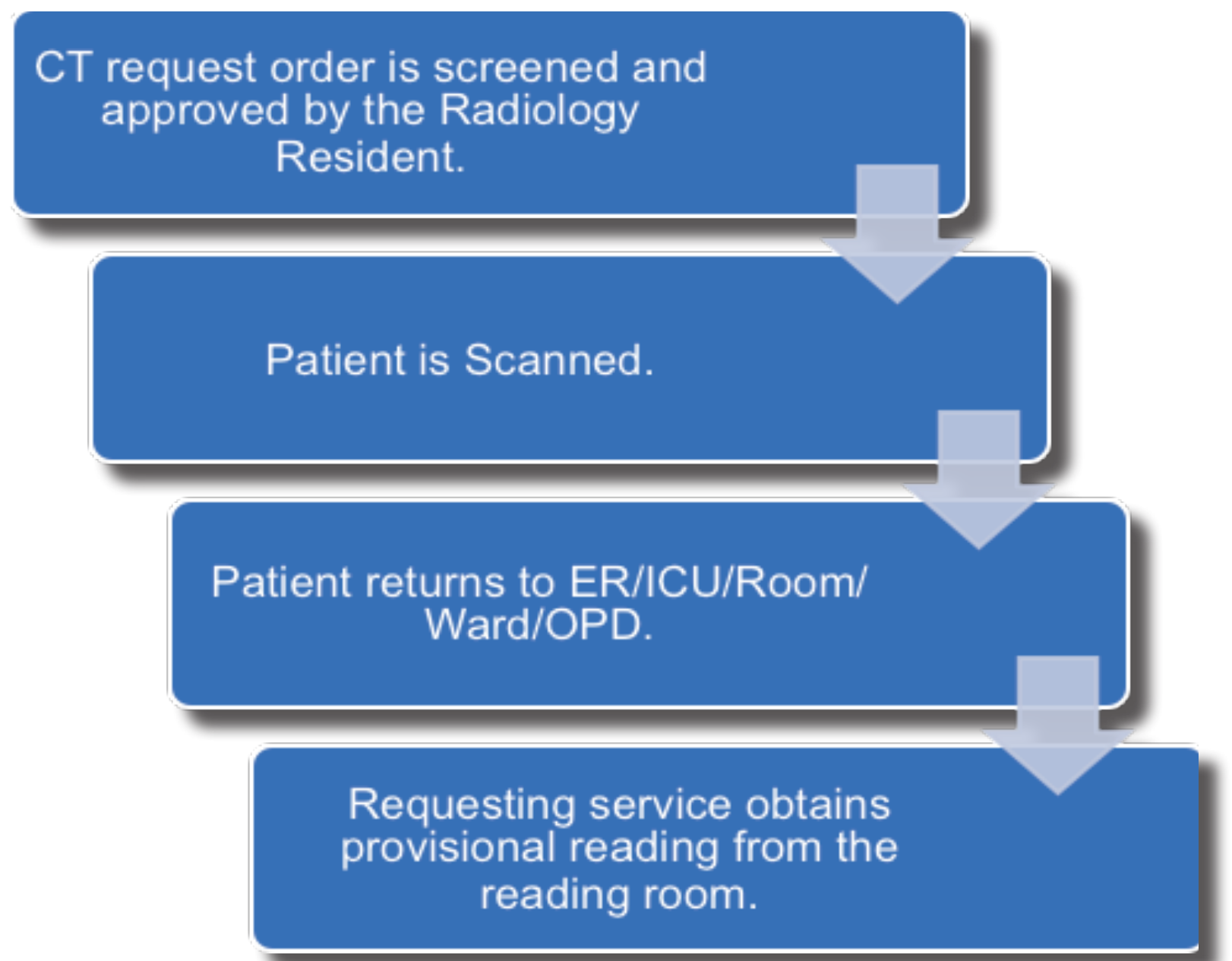

Figure 2. CT Scan Procedure in The Philippine General Hospital

The following time durations were recorded: from the point of CT order to scan (OS time), time from scan to interpretation (SI time) and interpretation to eventual relay (IR time) to the primary health care team. Total communication time was thereafter computed.

The mean, median, standard deviation, maximum and minimum values of these relevant time points were measured. A data collection sheet allowed the tracking of elapsed times between phases of the communication process and also identified possible reasons for delayed communication. Percentage compliance to the standard was thereafter computed. 


\section{RESULTS}

There were 684 cranial studies done from 4 February to 5 March 2019. Seventy-nine (79) critical findings were recorded, reported and relayed, accounting for $8.66 \%$ of the total during that 30 -day period. Accomplishment of the protocol using our key outcome measures was tabulated (Table 1). Of the 79 patients identified, there was $100 \%$ (79/79) compliance with delivery of critical radiology findings to the primary care team. There was $97.5 \%$ (77/79) compliance with the set time standard of $60 \mathrm{~min}$ utes from identification to direct communication (IR time). Two (2) cases out of the 79 identified critical findings were not relayed within 60 minutes because the ordering physician or nurse-on-duty could not be reached. Eventual response from the nurse-on-duty for both cases completed relay of these findings. This made up $0.03 \%$ of our total critical findings relayed.

Table 1. Time elapsed between phases of communication

\begin{tabular}{|c|c|c|c|c|}
\hline Time phase & Patient location & $\begin{array}{c}\text { Mean (SD) } \\
\text { (minutes) }\end{array}$ & $\begin{array}{l}\text { Median } \\
\text { (minutes) }\end{array}$ & $\begin{array}{c}\text { Range } \\
\text { (Minimum to Maximum) } \\
\text { (Minutes) }\end{array}$ \\
\hline \multirow{3}{*}{$\begin{array}{l}\text { Request/Order to scan } \\
\text { (OS) }\end{array}$} & ALL & 155.09 (134.43) & 98.00 & 7.00 to 551.00 \\
\hline & ER / ICU (Emergency) & $148.50(131.90)$ & 75.00 & $\begin{array}{l}7.00 \text { to } \\
551.00\end{array}$ \\
\hline & Wards / floors (Routine) & $180.44(146.56)$ & 136.50 & 13.00 to 476.00 \\
\hline \multirow[t]{3}{*}{$\begin{array}{l}\text { Scan to interpretation } \\
\text { (SI) }\end{array}$} & ALL & $46.54(44.01)$ & 31.00 & 1.00 to 189.00 \\
\hline & ER / ICU (Emergency) & $\begin{array}{c}47.40 \\
(41.60)\end{array}$ & 33.00 & $\begin{array}{l}1.00 \text { to } \\
189.00\end{array}$ \\
\hline & Wards / floors (Routine) & $\begin{array}{l}44.83 \\
(53.42)\end{array}$ & 26.50 & $\begin{array}{l}4.00 \text { to } \\
179.00\end{array}$ \\
\hline \multirow[t]{3}{*}{$\begin{array}{l}\text { Interpretation to relay } \\
\text { (IR) }\end{array}$} & ALL & $\begin{array}{c}9.13 \\
(12.04)\end{array}$ & 5.00 & 0.00 to 57.00 \\
\hline & ER / ICU (Emergency) & 9.05 (11.29) & 5.00 & 0.00 to 45.00 \\
\hline & Wards / floors (Routine) & $9.39(14.60)$ & 5.00 & 0.00 to 57.00 \\
\hline
\end{tabular}

Relevant time points in the whole communication process arranged from beginning to end were recorded, the most important being time of CT scan Order approval to actual Scan (OS time), from Scan to critical findings Interpretation (SI time), and from Interpretation to final Relay of critical result (IR time). These are listed below.

The mean OS time was $155.09 \pm 134.43$ minutes (median 98 minutes). The mean SI time was 46.54 \pm 44.01 minutes (median 31 minutes). The average time elapsed from interpretation to relay (IR time) to a member of the primary care team was $9.13 \pm$ 12.04 minutes (median 5.0 minutes). Finally, the average time it took from $\mathrm{CT}$ request approval to direct acknowledgement of critical findings (end to end) was $214.12 \pm 142.71$ minutes (median 31 minutes). Total communication time from point of detection of critical finding, were well within the set standard of 60 minutes.

Patient data was classified on presumed urgency of 
requests based on their locations (Table 1, Figure 2). Emergency room (ER) and intensive care unit (ICU) patients were grouped together and designated "emergency", while patients from the floors/wards were grouped together and identified as "routine." The breakdown of times is presented in the same table.
Out of the 79 critical findings identified, a total of 60 patients came from the emergency category-49 from the emergency room and 11 from the ICU. Only nineteen (19) patients were from the floors or wards, a ratio of approximately 3:1 (Figure 3).

\section{Critical Findings Relayed Based on Location}

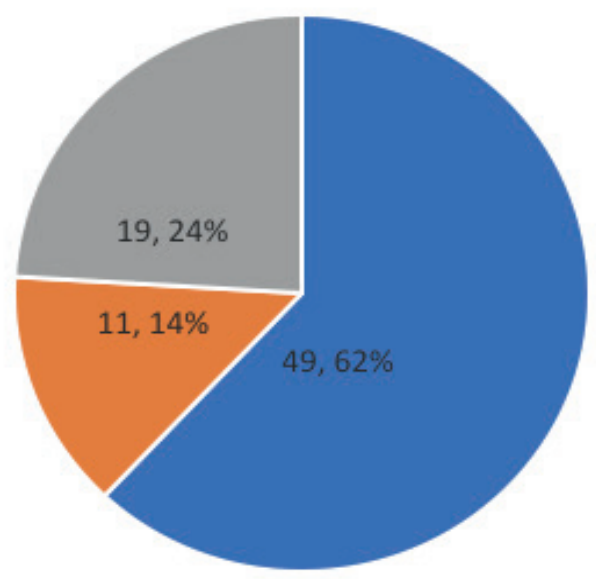

\section{- Emergency Room " Intensive Care Unit Patients "Floor/ Ward Patients}

Figure 3. Critical Findings Based on Location

Emergency and ICU patients were scanned sooner, and results relayed faster than routine patients. Their average OS time was 148.50 ( \pm 131.90$)$ minutes, average SI time was $47.40( \pm 41.60)$ minutes and average IR time was $9.05( \pm 11.29)$ minutes. With the routine studies, the average OS time was 180.44 ( \pm 146.56$)$ mins, average SI time was $44.83( \pm 53.42)$ mins and average IR time was $9.39( \pm 14.60)$ minutes. Finally, the average order to relay time was 207.85 $( \pm 141.25)$ minutes for emergency patients and 234.67 $( \pm 149.62)$ mins for routine patients respectively.

\section{DISCUSSION}

The Joint Commission (TJC) is explicit in stating the need for timely identification, recording and effective relay of critical findings of tests and diagnostic procedures. In this regard, TJC included in its 2011 National Patient Safety Goals, effective communication between healthcare providers, by mandating them to record and communicate critical findings in tests or laboratory procedures in a timely basis. Radiologists share the responsibility of relaying critical findings to those responsible for immediate management [9]. Significant delays in communication of critical findings can lead to a cascade putting the patient at greater risk of morbidity or death.

Our study showed that by following a standardized communications protocol, timely relay of critical findings is possible in our institution. Our results exhibited excellent compliance with the standard safe practice recommendations of the Massachusetts Hospital Association and the Coalition, with a 100\% (79/79) success rate in delivering critical findings 
to the primary care team and having $97.5 \%$ (77/79) compliance to the recommended 60-minute standard. The two (2) non-compliances were not communicated within 60 minutes from identification because the primary requesting physician and nurseon-duty were not answering their phones despite several attempts. The findings were eventually relayed beyond the 60-minute limit.

Our data collected compared favorably with a study done by Honig et al., which assessed the timing of critical findings notification in neuroradiology. In this 2013 study, they surveyed the different relevant communication time points between radiologists and caregivers during an 8-week period [11]. The mean time from critical findings discovery to referring physician notification was $8.8 \pm 6.9$ minutes [11]. In our study, the average for the same parameter (IR time) for all patients was $9.13 \pm 12.04$ minutes. Comparing values gathered from ER/ICU and floor patients, we found that order/request to scan (OS) times of emergency patients were faster than routine patients. Subsequently, there were also small time differences between the 2 subgroups. The average IR times in the two groups, $9.05( \pm 11.29) \mathrm{min}$ utes and $9.39( \pm 14.60)$ minutes, respectively, further demonstrated this. Cumulatively, the time it took to complete the process was faster for the ER/ICU patients (Table 1). In our study, the time savings from each step resulted in a total difference of approximately 30 minutes between ER/ICU patients and routine patients. This is not unexpected as patients from these locations are given the earliest possible schedules and their scans are interpreted first. The smallness of the difference, however, does show that patient location or disposition did not appreciably affect the time involved in delivery of critical findings to the requesting physician.

With the exception of 2 cases, time values across all relayed critical findings met the 60 -minute recommendation for "red zone" values being practiced in the Department of Radiology in the Brigham and Women's Hospital in Boston. Overall, the findings are congruent with the results of other studies, specifically Honig et. al., which exhibited even higher time intervals. In their study, the mean time elapsed between study order to study completion was 7 hours and 3 minutes (compared to our mean of 155.09 minutes or approximately 3 hours).

In spite of the relative speed, 3 hours average between study order and completion is still not as fast as the referring physicians would like. These may be explained by several factors experienced during the study, including varying load during the work day and having only one working CT scan machine. That is, all emergency and routine cases were done on a single CT scan machine. Nonetheless, the times obtained showed remarkable comparability to the results of other similar studies monitoring timing of critical findings relay as seen in Honig et al. and Valenstein et al's works [8]. The gathered data reflect that overall, communication and notification of critical results operated within the ideal recommended standard.

This study acknowledges the limitation of the 30day sampling duration. It is recommended that similar or follow-up studies extend the study duration to further validate the general trends and the other possible sources of communication delay. We cannot discount the possibility of bias of the participating physicians in this study. Because participants were aware of the on-going study, they may have been more compliant and conscientious in recording and relaying critical findings, artificially improving notification times. With a longer study duration, this bias may be reduced. Testing statistical significance between times of different groups (emergency and ICU versus routine) in future studies is suggested to further validate results.

All means of communication done during this study were through the initiative of the radiologists involved. In the Philippine General Hospital, the centralized communication system is not designed to relay clinical information and will, at best, inform a specific care provider to respond to a request. There were instances when the requesting physician and even the nurse on duty could not be contacted, leaving the burden to communicate critical findings on radiologists through their own means. We recommend back-up routes of communication to facilitate immediate notification of the primary 
care team and the review and improvement of the communications architecture of the hospital to help physicians implement the critical findings protocol. This study recommends the use of a thoroughly vetted, multidisciplinary approved radiologic critical findings, in neuroradiology and other systems. This would greatly help improve identification and communication of critical findings. We further recommend that follow-up studies test a multidisciplinary critical findings protocol to include a more comprehensive critical findings list.

In future studies, critical findings noted by the resident radiologist should be verified by a more senior member of the team for correctness of findings. Our study relied on the accuracy of the preliminary reading of the senior resident on call, without further validation. Follow-up studies may opt to check the accuracy of these critical findings.

\section{CONCLUSION}

The times obtained in this study serve as baseline data for time-motion studies of critical relay findings. The times were well within standards and comparable to times obtained in other institutions abroad. Effective communication of critical findings using a protocol adopted from set standards of safe practice recommendations is achievable in our institution. The proposed protocol exhibited compliance to and performed well against established benchmarks. The timely identification and delivery of critical findings to the primary care team is central to patient management and should be practiced in our setting. Identified delays in specific time points of communication were primarily due to external factors not controlled by the radiologist (unanswered calls and lack of central communication system). Despite these time delays, prompt critical findings identification and communication were still possible and achievable. Findings in this study should be used to develop other critical findings protocols not limited to neuroradiology, which will only improve patient safety and outcomes in any institution.

\section{CONFLICTS OF INTERESTS}

The authors declare that there is no conflict of interests. 
[1] Leonard M, Graham S, Bonacum D. The human factor: the critical importance of effective teamwork and communication in providing safe care. Quality \& safety in health care. 2004; 13(Suppl 1): i85-i90. doi:10.1136/ qshc.2004.010033.

[2] Dingley C, Daugherty K, Derieg MK, et al. Improving Patient Safety Through Provider Communication Strategy Enhancements. In: Henriksen K, Battles JB, Keyes MA, et al., editors. Advances in Patient Safety: New Directions and Alternative Approaches (Vol. 3: Performance and Tools). Rockville (MD): Agency for Healthcare Research and Quality (US); [Online] 2008 Aug. [Cited 2019 August] Available from: https://www.ncbi.nlm.nih.gov/books/ NBK43663/

[3] Woolf S. H., Kuzel A.J., Dovey S. M., et al. A String of Mistakes: The Importance of Cascade Analysis in Describing, Counting, and Preventing Medical Errors. Annals of Family Medicine. 2004; 2(4):317-326 DOI: 10.1370/afm.126.

[4] ACR practice parameter for communication of diagnostic imaging findings. [Online]. 2014 [Cited 2018 August]. Available from https://www.acr.org/ClinicalResources/Practice-Parameters-and-Technical-Standards/ Practice-Parameters-for-Documentation-and-Reporting.

[5] Gershanik EF, Lacson R, Khorasani R. Critical finding capture in the impression section of radiology reports. AMIA
Annu Symp Proc. 2011; 2011:465-469.

[6] The Joint Commission. About Us [Online]. 2018 [Cited 2018 August] Available from https://www.jointcommission. org/about_us/about_the_joint_commission_main.aspx.

[7] Khorasani R. Optimizing communication of critical test results. J Am Coll Radiology. 2009; 6(10): 721-723. DOI: 10.1016/j.jacr.2009.07.011

[8] Viertel V.G., Trotter S.A., Babiarz L. S., et al. Reporting of Critical Findings in Neuroradiology. AJR American Journal of Radiology. 2013; 200(5):1132-1137 DOI:10.2214/ AJR.12.9041

[9] Hanna D., Griswold P., Leape L., et al. Communicating Critical Test Results: Safe Practice Recommendations. Joint Commission Journal on Quality and Patient Safety. 2005; 31(2): 68-80.

[10] Trotter SA, Babiarz LS, Viertel VG, et al. Determination and communication of critical findings in neuroradiology. J Am Coll Radiol. 2013;10(1):45-50.

[11] Honig SE, Honig EL, Babiarz LB, et al. Critical Findings: Timing of Notification in Neuroradiology. AJNR Am J Neuroradiol. 2014; 35(8):1485-1492. DOI: https://doi. org/10.3174/ajnr.A3918 